Research Article

\title{
Effect of Supplementary Branch Chain Amino Acids on the Ability of Table Tennis Players by Image Analysis of Visual Sensors
}

\author{
Jianqiong Jiang (10) \\ College of Physical Education, Sichuan Minzu College, Kangding, 626001 Sichuan, China \\ Correspondence should be addressed to Jianqiong Jiang; 140382@scun.edu.cn
}

Received 20 August 2021; Revised 6 October 2021; Accepted 12 October 2021; Published 8 November 2021

Academic Editor: Haibin Lv

Copyright (c) 2021 Jianqiong Jiang. This is an open access article distributed under the Creative Commons Attribution License, which permits unrestricted use, distribution, and reproduction in any medium, provided the original work is properly cited.

\begin{abstract}
The purpose of this article is to in-depth explore the specific effects of supplementation of branched-chain amino acids on table tennis performance and its mechanism. Athletic ability refers to the ability of a person to participate in sports and training. It is a comprehensive manifestation of a person's physical form, quality, function, skills, and psychological abilities. From a biochemical point of view, the level of exercise capacity mainly depends on the energy supply, transfer, and utilization of energy during exercise. The metabolic basis of exercise capacity and its influencing factors, the central and peripheral causes of sports fatigue, and the general law of the recovery of consumed substances after exercise provide a theoretical basis for athletes' selection of talents, evaluation of training effects, and formulation of training plans. The main function of the vision sensor is to obtain enough of the most primitive images to be processed by the machine vision system. Use the latest vision sensor technology for in-depth research. This article takes 20 professional table tennis players with the same sports score as the research object and divides the athletes into the control group and observation group supplemented with branch chain amino acids. For the observation group, the exercise plan is to design two one-hour table tennis games, divided into two days, with a 10-minute break after half an hour of the game, and the athletes in the observation group to have a weight of $0.3 \mathrm{~g} / \mathrm{kg}$ before and during the rest of the game. Weight measurement supplemented with branched-chain amino acids, the control group, athletes supplemented with the same amount of ordinary mineral water, the athletes' fatigue index, endurance index, and reaction ability index were scored, and the athlete's blood was drawn to test the sports-related biochemical indicators after the end of table tennis. The results of the study showed that after supplementation with branched-chain amino acids, the fatigue index of the athletes in the observation group was $(7.5 \pm 1.2)$, the fatigue index of the control group was $(11.2 \pm 1.5)$, the endurance index of the observation group was $(28.4 \pm 4.8)$, that of the control group was $(19.6 \pm 3.7)$, the reaction ability index of the observation group was $(18.4 \pm 4.2)$, and the control group was $(12.3 \pm 2.8)$. At the same time, the athletes' plasma antioxidant capacity and metabolism level of the observation group were significantly higher than those of the control group. Therefore, it can be seen that supplementing branched-chain amino acids can effectively improve the athletic ability of table tennis players.
\end{abstract}

\section{Introduction}

The vision sensor appeared in the late 1950s and developed very rapidly. It is one of the most important sensors in robots. Robot vision began to deal with the world of building blocks in the 1960s and later developed to deal with indoor scenes such as tables, chairs, and lamps and then deal with the real world outside. Table tennis is based on other forms of aerobic exercise and supplemented by other forms of anaerobic exercise. Table tennis is very antagonistic, and athletes often need to consume a large amount of comprehensive ability and a large amount of physical reserve every day to successfully complete the game. With the continuous increase of human exercise intensity and the continuous increase of physical energy consumption, athletes will gradually produce some exercise fatigue.

Amino acids and enzymes are the main raw materials for the metabolism and synthesis of various proteins in the body and are the main energy substances that are decomposed by oxidative cells in the body. The products of certain new 
amino acids or other enzyme metabolites are also a metabolic factor in the body [1]. Compared with human carbohydrate and human fat metabolism, amino acids are not the main source of energy metabolism in the metabolic process of aerobic exercise, but increasingly, research data has shown that aerobic exercise will continue to increase some amino acids, especially. It is said that it is the oxidation of some branched-amino acids in human muscle tissue [2]. Acute stroke and a certain concentration of serotonin in serum 5-6 can effectively delay the symptoms of central brain fatigue and improve and help improve the ability of football players [3].

To explore the specific effects and mechanisms of supplementation of branched-chain amino acids on table tennis performance and display and analysis of images using the visual sensor technology, this article consulted a large number of related materials. Among them, Kephart et al. gave an introduction to table tennis, emphasizing that China is a strong country in table tennis, and pointed out that table tennis is a sport that consumes a lot of energy and requires very high physical fitness and reaction ability of athletes [4-6]. Chen et al. proposed in his article that table tennis is a highly competitive sport. Athletes will have a great exercise load in this sport, which puts the content secretion system and respiratory system of the athlete in a high-load state, so athletes are prone to sports fatigue [7]. Yokota et al. elaborated on the constituent elements, chemical properties, and biological characteristics of branched-chain amino acids, emphasized the importance of branched-chain amino acids to human functions, analyzed the relationship between branched-chain amino acids and exercise, and discussed branched-chain amino acids and the mechanism of action of exercise [8]. Wambacq et al. found through relevant experimental research that branched-chain amino acids can improve the athletic ability of table tennis players and supplementation of branched-chain amino acids can delay athletes' exhaustion time and improve athletes' endurance and reaction speed and point out that for athletes, the supplement of branched-chain amino acids is essential [9]. Parivash et al. found through research that branchedchain amino acids can effectively reduce the heart rate of humans or animals during exercise, improve blood antioxidant effects, and accelerate body metabolism; at the same time, it emphasizes that excessive consumption of branched-chain amino acids will have certain side effects [10].

Among these research scholars, they have been pursuing how to reduce the fatigue time of athletes and how to quickly restore their energy, to ensure that they can ensure that their level does not drop currently. However, due to the lack of experimental technology, there is no actual data to support their research and the consideration is not comprehensive enough.

In the research on the specific effects of supplementation of branched-chain amino acids on table tennis performance and its mechanism, this article summarizes and analyzes the research experience and results of a large number of predecessors. In addition, this article has made some innovations in the research content and research methods and the specific innovations include the following two points: first, in the first half of the women's table tennis individual competition, this method of intermittent cumulative increasing and decreasing of load training can be used to obtain 20 women's table tennis players who have not accepted this test for a short period of time and high intensity. The best incremental load test value during interval incremental training is used to design the athlete's exercise volume in the experiment. Second, this article uses the multicomponent statistical data analysis method collected by data analysis software for the first time and uses TGSS130 software to collect and analyze the statistical data of athletes' blood physiological indicators after table tennis, which greatly improves the accuracy of the research results. Third, immediately after the end of each half of the test, the subjective feeling fatigue rating scale (RSE) is used to test the physical fitness of table tennis players and record the four levels of rising in real time. The desktop heart rate exercise monitor can detect the whole the heart rate of each athlete during the physical fitness test.

\section{Visual Sensor Analyzes Physical Training Methods and the Role of Branched-Chain Amino Acids}

2.1. Vision Sensor Technology. The vision sensor is the direct source of information for the entire machine vision system. It is mainly composed of one or two graphic sensors, sometimes with light projectors and other auxiliary equipment. The vision sensor has thousands of pixels that capture light from an entire image. The clarity and fineness of an image are usually measured by resolution, expressed in the number of pixels. Some of the vision sensors provided by Banner Engineering can capture 1.3 million pixels. Therefore, the sensor can "see" a very delicate target image even if it is a few meters or a few centimeters away from the target. The main function of the vision sensor is to obtain enough original images to be processed by the machine vision system. The image sensor can use a laser scanner, a linear and area CCD camera, or a TV camera or the latest digital camera. Visual sensing technology is one of the seven categories of sensing technology. The visual sensor refers to the following: calculate the feature quantity of the object (area, center of gravity, length, position, etc.) by processing the image captured by the camera and a sensor that outputs data and judgment results. Figure 1 shows a small machine vision system that integrates image collection, image processing, and information transmission with vision sensor technology.

Vision sensor technology is widely used in our daily life. Its typical application framework is shown in Figure 2. Vision sensors have many different communication interfaces, which can communicate with some external hardware devices through TCP/IP, CAN, OPC, and other buses. It can exchange data and information, and can also connect to robot controllers and PCs and other devices. The smart vision sensor under the smart vision sensing technology is also called a smart camera, and it is a new technology that has been developing the fastest in the field of machine vision in recent years. The smart camera is a small machine vision system with the functions of image acquisition, image 


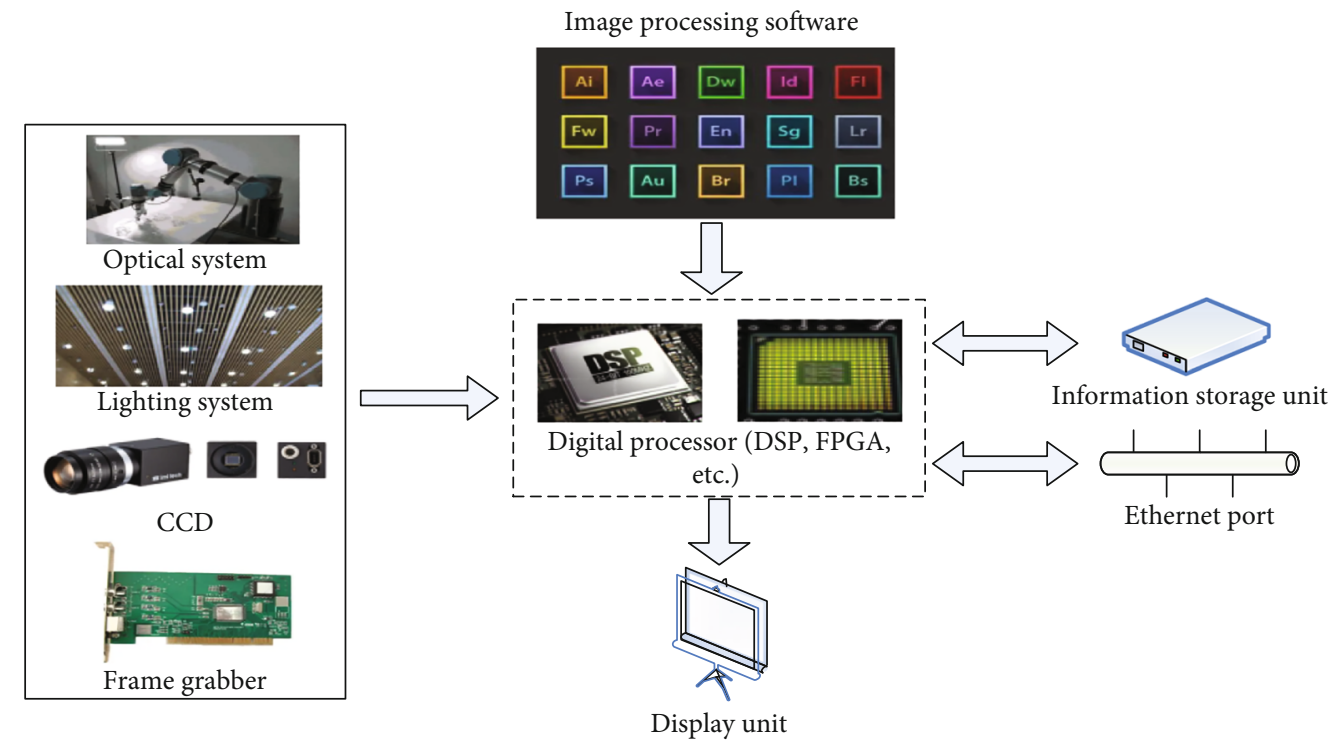

FIGURE 1: Sensor technology and image processing technology.

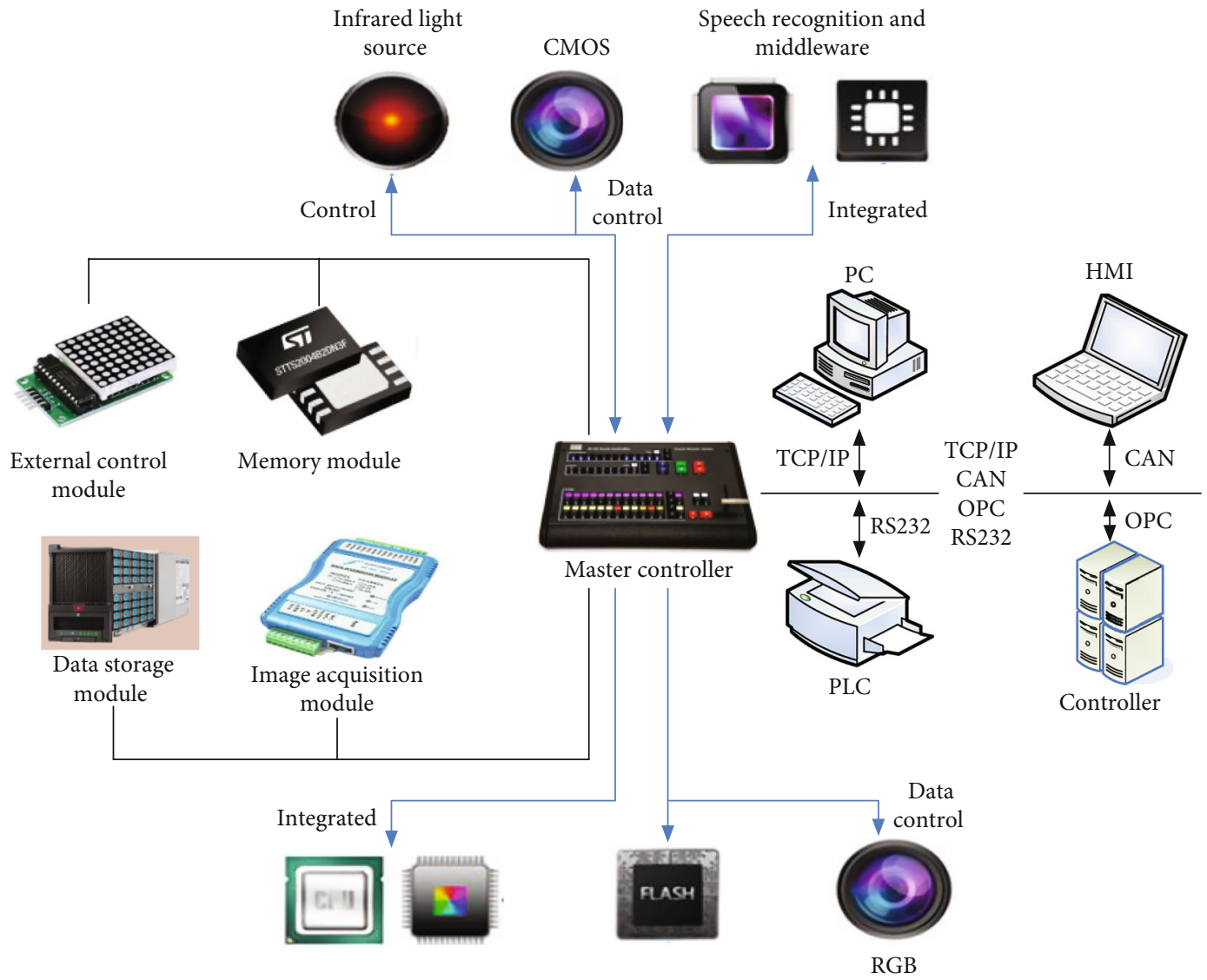

Figure 2: Typical application framework based on vision sensors.

processing, and information transmission. It is an embedded computer vision system. It integrates image sensors, digital processors, communication modules, and other peripherals into a single camera. Due to this integrated design, the com- plexity of the system can be reduced and the reliability can be improved. At the same time, the system size is greatly reduced, which broadens the application field of vision technology. 


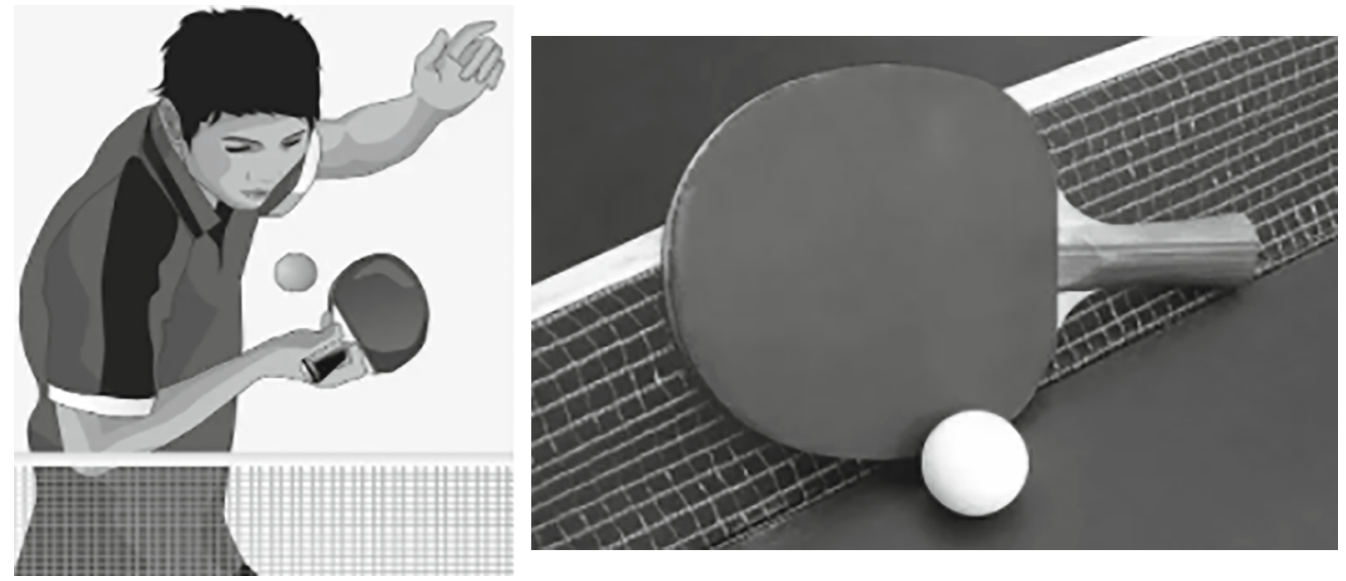

Figure 3: Schematic diagram of table tennis.

2.2. Methods of Supplementing Physical Fitness in Table Tennis. Table tennis combines high IQ and high health. That needs athlete training. In order to correctly judge the reaction speed during exercise, athletes need to maintain a high degree of concentration and sensitivity, but always in a state of high tension, it is easy to cause central nervous system fatigue [11]. Figure 3 shows a schematic diagram of table tennis. Supplementing branched-chain amino acids reduce the speed of tryptophan entering the brain, reduces the production of serotonin, maintains the normal excitability of the brain, and slows the appearance of central fatigue [12]. Supplementing branched-chain amino acids can reduce the concentration of ammonia in the blood and prevent and reduce the toxic effects of ammonia. Branched-chain amino acids can also be converted into acetic acid, and turbine is an important nutrient necessary for the human body. Its main biological function is to maintain the stability of the membrane structure, maintain the balance of osmotic pressure, prevent cell membrane lipid peroxidation, relieve lipid peroxidation caused by exercise, and promote the enhancement of the body's antioxidant enzyme activity after exercise. Exercise steel is prevented by low cell function, thus delaying the onset of fatigue. The branched-chain amino acids are restored to a certain degree. Doing intense exercise can metabolize the protein in the body and promote the synthesis of tissues, which is very suitable for the recovery of table tennis players' physical strength.

In the table tennis shooting game, the players' offensive and defensive speed conversion is quite fast, especially to highlight the player's fastest backhand strike speed. This inevitably requires football players to pay attention to a high degree of concentration in technical training and sports games. Therefore, the daily dietary conditioning of athletes should be able to provide us with the corresponding food nutrition. In particular, it is high in protein, sugar, and high fat. They all play an important comprehensive role in our daily life and cultural activities. Table tennis is indispensable in ball games. It is small in size, fast in running speed and high in flexibility. Nutritional activity refers to the whole physiological process of the human body through absorbing, digesting, and rationally using various foods and various nutrients and minerals in it to maintain the normal activities of human life. There must be a close interrelationship between nutrition and human exercise and metabolism. It can provide a variety of nutritional energy that is not necessary for normal human exercise and promote the digestion and synthesis of the basic components of a new generation of tissue cells. It is for repairing human tissue cells and regulating functions, an important raw material for the metabolic process of human exercise. The law of balanced diet requires that the football players' daily exercise diet is balanced and nutritious. Scientifically monitor and control the diet of athletes to conduct regular physical examinations, determine whether each athlete lacks nutrition in time, adjust and understand the athlete's diet in time, and improve the athletes' daily eating habits by combining diet control and supplementing athletes' nutrients. Athletes can perform at a good level, actively participate in training and competitions, and achieve excellent results. Figure 4 shows the detailed flow chart of the table tennis game. In Figure 4, we can clearly see that the winner is determined through layers of competition but the winning side needs to constantly accept challenges from other people. During this time, the athlete's energy cannot be in the best state, so it is necessary to supplement this energy.

In table tennis, fast exercise and explosive whiplash are the main forms of sport. Therefore, the metabolism of table tennis combines aerobic and anaerobic metabolisms. Aerobic metabolism is the basis and anaerobic metabolism is the main metabolism. In the daily diet, we should pay attention to the supplement of protein and vitamin $\mathrm{B}$ and choose foods that help to quickly restore phosphoric acid [13]. At the same time, to prevent muscle cramps caused by calcium deficiency, athletes need to pay attention to the absorption of calcium, vitamin $\mathrm{C}$, and vitamin $\mathrm{D}$ (vitamin $\mathrm{C}$ promotes calcium absorption). Second, the stability of the internal environment is indispensable for athletes to maintain a clear mindset during training and competition. Foods with high nutritional value contribute to the stability of the internal environment. In table tennis, athletes need to pay close attention to their eyesight, so vitamin A supplements are needed. Table tennis players also need to supplement 


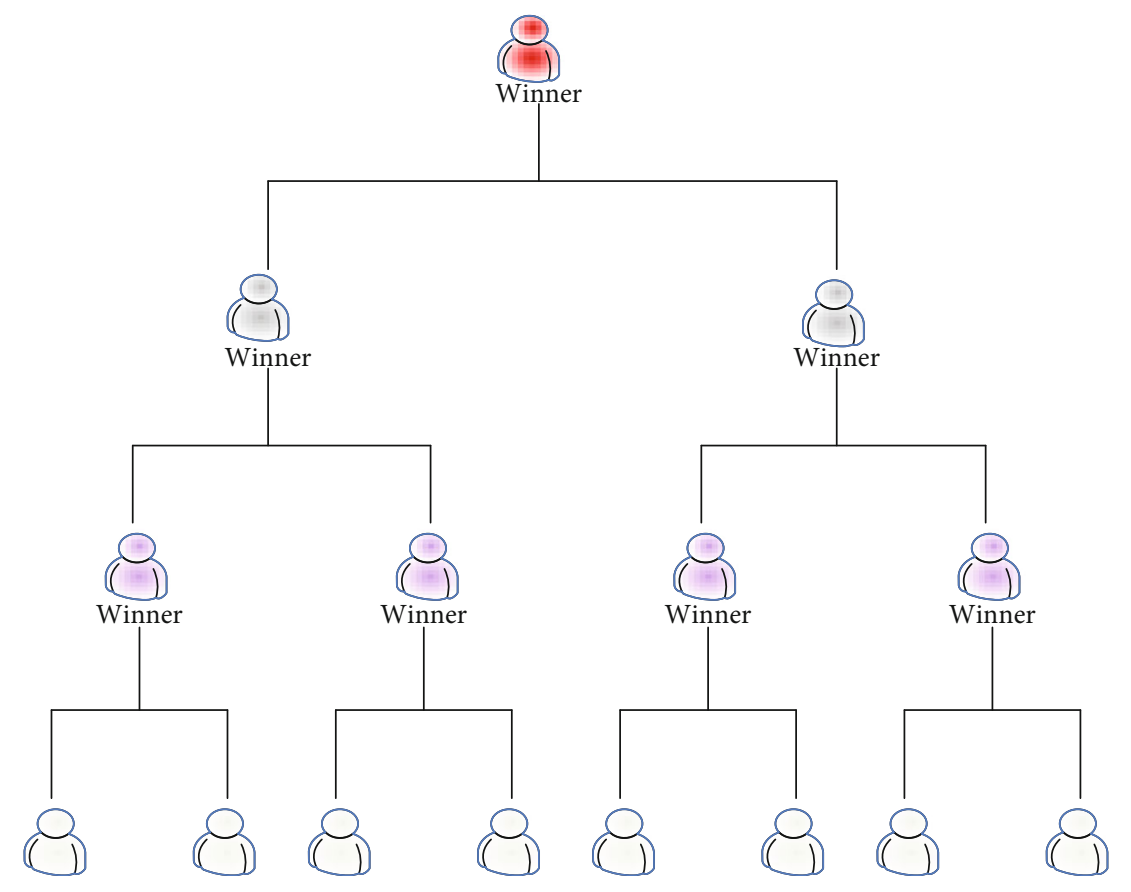

FIgURE 4: Detailed flowchart of table tennis competition.

calcium, iron, zinc, and other minerals. To promote calcium absorption, vitamin $\mathrm{D}$ and magnesium need to be supplemented at the same time. Calcium must be the main supplement of the diet. Generally speaking, it is recommended to drink soy milk for breakfast, milk for lunch and dinner, and milk before going to bed. Supplement nutrition in time, promote the recovery of muscle glycogen, and promote the growth and repair of skeletal muscle. The ideal protein supplement needs to reach the peak protein requirement within 70 minutes after exercise. Fat is not only an important source of energy supply for table tennis players; it is also an important source of energy supply for other sports players. Too much fat will reduce the quality of athletes' endurance, which affects the performance of skills and tactics. Therefore, the nutritional intake should be reasonably arranged according to the athlete's diet and intake.

\subsection{The Role and Mechanism of Branched-Chain Amino} Acids in Exercise. Amino acid itself is an organic compound with special scientific significance, because most of them are closely related to human life cycle activities. It is the basic functional composition control element of proteins, and it is indispensable or lacking nutrient to maintain human health. Amino acids can provide a variety of energy in human sports and are closely related to the ability of human athletes. The biosynthesis and metabolism of amino acids in the branched chain are limited to certain plants and specific types of microorganisms. It is almost a daily necessity for all humans. Amino acids need to be obtained through various food exercises [14]. The amino acid on the branched chain is usually an amino acid with a carbon atom skeleton on the branched chain. The amino acids in the branched chain are mainly dissimilated by fat in the leg muscles and are easily oxidized by fat to provide more energy. The oxidore- ductase can transfer the two amino groups to the carboxylic acid molecule to obtain acetylacetonate and glutamic acid, which react to form hydroxylamine and hydroxy glutamic acid. Tyrosine lactones are the most easily oxidized by the human body among all branched-chain amino acids. After prolonged strenuous exercise, the protein energy of muscles and internal organs will decrease, accounting for up to $10 \%$ of the total energy. Figure 5 shows the role of branchedchain amino acids in the body's exercise process, which is of great benefit to the human body. It can increase the rate of protein synthesis and reduce the rate of protein degradation.

For this endurance exercise, the metabolic process of branched-chain myocardial amino acids taken by the muscles during exercise only affects the muscle recovery after endurance exercise but has no effect on the human muscle cell IS that is concentrated during the exercise recovery process. Through various experimental observations and with the support of experimental data, therefore, it is concluded that the results are based on the quantitative determination of muscle protein and its metabolic catalytic products, because muscle TYR and muscle PHE synthesized by it cannot be broken down by all muscle fat cells located in the human body or by enzymes. Therefore, TYR and the released human muscle and synthetic PHE have been broken down into new muscle cell proteins [15]. However, in an endurance aerobic exercise, supplementing a large amount of branchedchain intermediate amino acids prevented a large increase in Tyr and PHS in the blood of muscles and liver, which also directly reflected that the large intake of branched-chain intermediate amino acids would be severely reduced. It prevents the rapid hydrolysis of proteins in blood and muscle blood. During a long or short period of moderate speed exercise, due to the large amount of glycogen stored in the kidney 


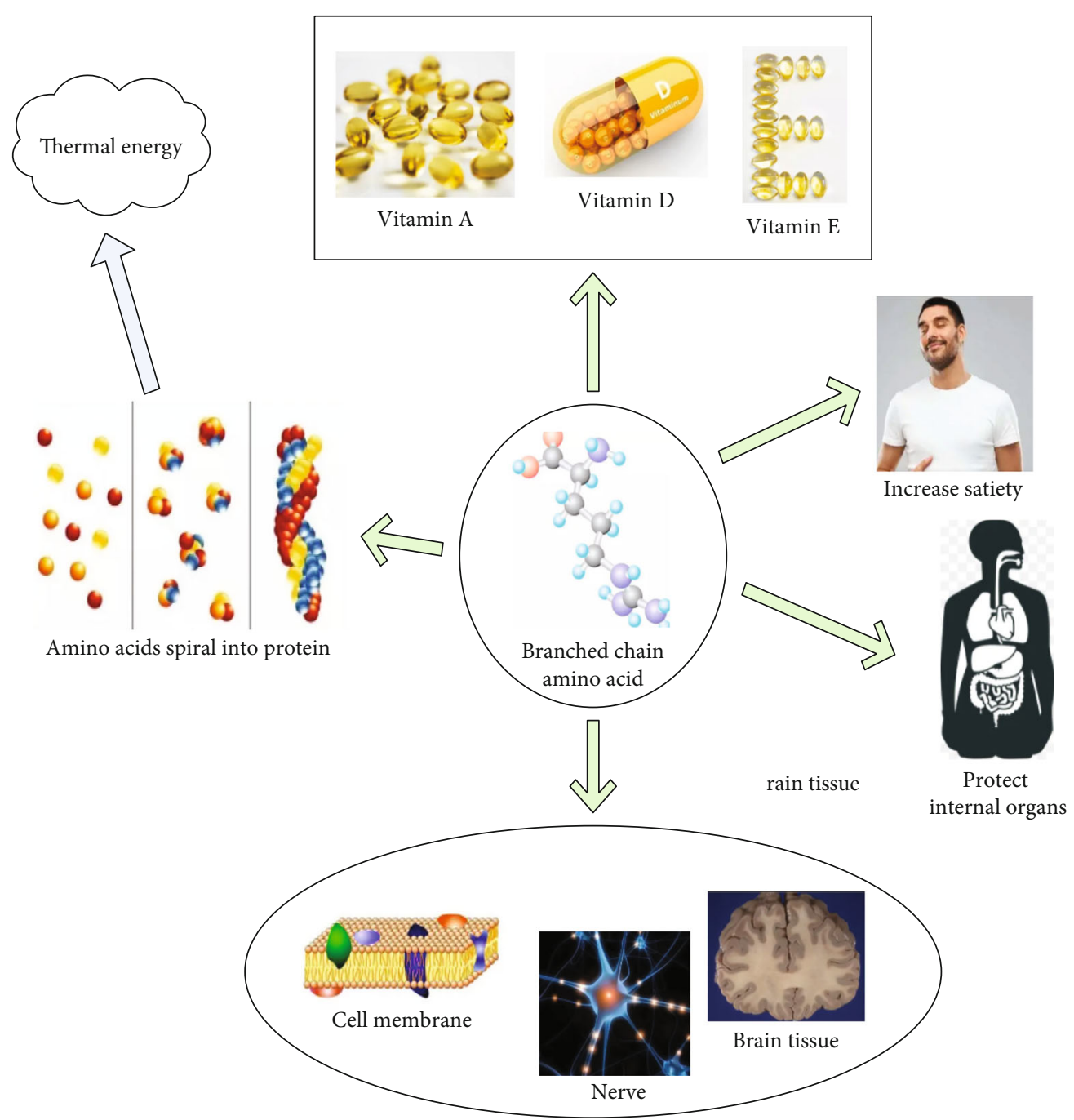

Figure 5: The role of branched-chain amino acids in human exercise.

and liver, the blood glucose level will drop, which may be a known pathogenic mechanism that will seriously affect the central nervous system. . The sports center is another important cause of central fatigue in that it is the central neurotransmitter (especially when it is 5-hexahydroxytryptamine $(5-\mathrm{Ht}))$ that increases the hormone release rate in the brain. Abnormal changes in the level of the 5-ht hormone in the brain may directly affect people's sleep, waking up, and controlling of emotions [16]. Tryptophan hydroxylase is the only the first step to catalyze the synthesis of the 5-ht substrate. Since the synthesis of tryptophan hydroxylase is not saturated by substrate synthesis, the rate of change in the substrate synthesis of 5-ht affects the synthesis of color in human blood. The obvious changes in the concentration of amino acid molecules are sensitive to the number of molecules transported by synthetic tryptophan, across the capillary and brain nerve barrier. And because the same amount of tryptophan transport and synthesis is also greatly restricted by the transport synthesis capacity of the entire trans-vascular and brain central barrier gene transport synthesis carrier system, and because tryptophan and a large number of other neutral branched-chain amino acids (including branched-chain amino acids). Neutral amino acids share the same in the entire trans-blood-brain barrier synthetic transport carrier system. Therefore, because tryptophan and other branched-chain neutral amino acids coexist in the competition mechanism of different transport amounts, the distribution percentage of tryptophan transport concentration is also an important factor that directly affects the rate of 5-ht acid synthesis and transport.

Branched-chain amino acids are the only one in the main metabolism of amino acids in muscle. In continuous exercise, branched-chain amino acids are continuously consumed by muscle cells and the concentration of branched-chain amino acids in the blood decreases, which also means that the concentration of free tryptophan in plasma increases. Exercise increases the ratio of free tryptophan and branched-chain amino acids (Try/branched-chain amino acids) in the plasma, promoting the delivery of tryptophan to the brain and the synthesis of 5-HT, which ultimately leads to central fatigue. Figure 6 shows the mechanism of branched-chain amino acids in exercise. Intake of branched-chain amino 


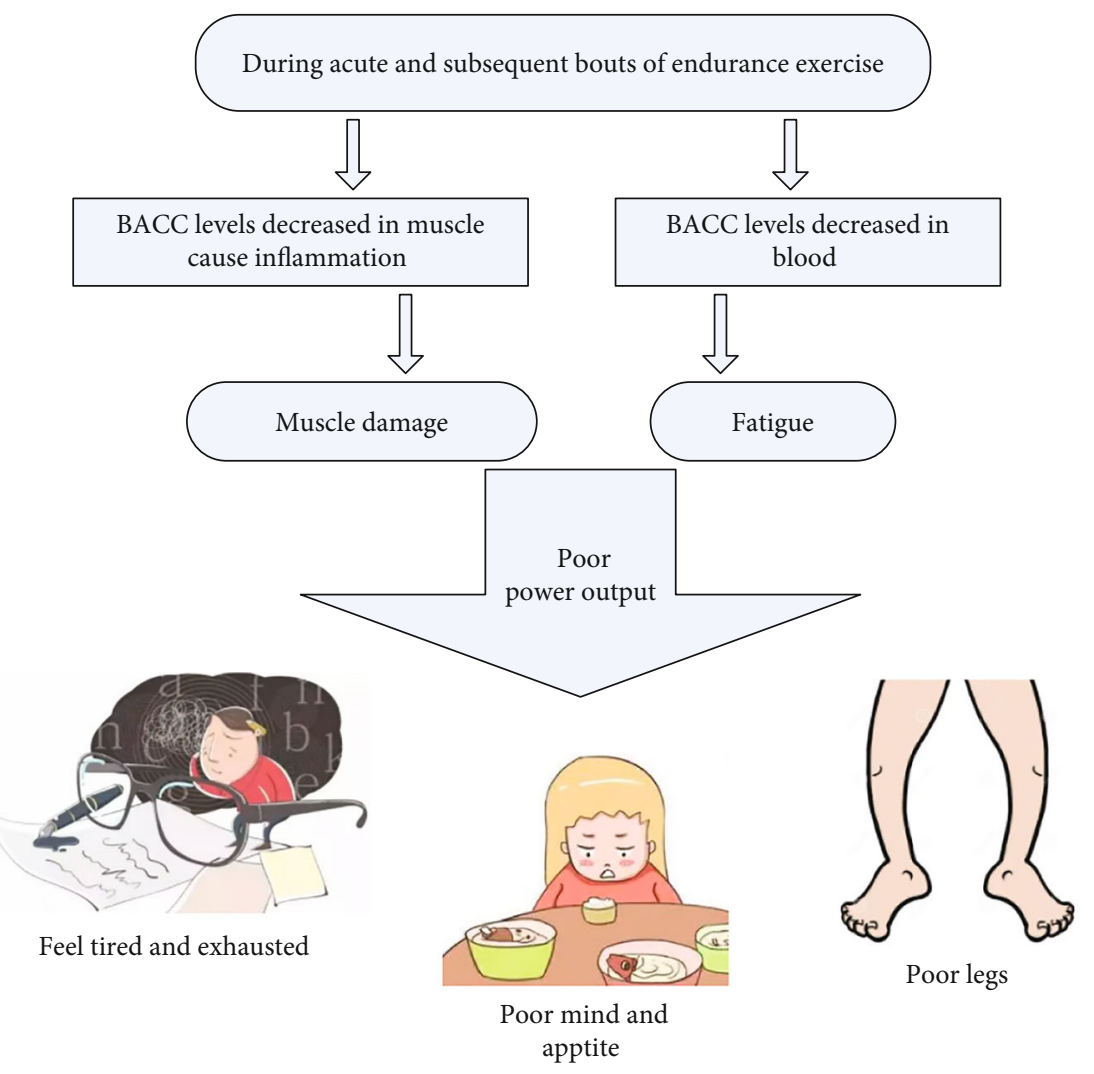

Figure 6: The mechanism of branched-chain amino acids in exercise.

acids can increase the concentration in plasma and maintain the balance of try/branched-chain amino acid increase caused by exercise, thereby reducing the synthesis and release of 5-HT, reducing central fatigue, and supplementing branched-chain amino acids, and carbohydrates can improve the mental agility of athletes [17]. Some research results have shown that before exercise or sports nutrition, the concentration of partial chain amino acids in the blood increases. The reason for these results may be the number of partial chain amino acids and the intake of different chain amino acids $(10-20 \mathrm{~g})$. The higher ammonia concentration is possible, but in the case of exercise and recovery, a smaller amount of different chain amino acids (5-8g or $100 \mathrm{mgkg}$ weight) recurs and no intake is observed, but the amount of ammonia released by muscle increases. This nutritional supplement can increase the concentration of branched-chain amino acids in the plasma, thereby maintaining a balance between the increase in the concentration of branched-chain amino acids during and after exercise. Therefore, during long-term exercise, proper intake of branched-chain amino acids will not lead to blood ammonia concentration.

\section{Related Experiments on the Influence of Branched-Chain Amino Acids on Exercise Ability}

3.1. Selection of Table Tennis Players. This article takes 20 professional table tennis players with the same sports score as the research object, aged between 18 and 22, including 12 males and 8 females. The average height of men is $(175 \pm 5 \mathrm{~cm}$ and the average height of women is $(166 \pm 5 \mathrm{~cm})$, who have received professional table tennis training for more than 5 years. The athletes are divided into the control group and the observation group supplemented with branched-chain amino acids. The athletes in the observation group are measured at $0.3 \mathrm{~g} / \mathrm{kg}$ weight during the rest time before and during the game, supplemented with branched-chain amino acids and the control group athletes supplemented with the same amount of ordinary mineral water. The basic situation of the tested table tennis players is shown in Table 1. The sports venue is in the stadium, and the lives of each athlete participating in the experiment are under surveillance. For the authenticity of the experiment, their training is done all together.

\subsection{Design of the Exercise Program and Supplement of Branched-Chain Amino Acids}

3.2.1. Supplements of Branched-Chain Amino Acids. Before and during the competition, athletes in the observation group were given $0.3 \mathrm{~g} / \mathrm{kg}$ body weight of branched-chain amino acids, while athletes in the control group were given the same weight of ordinary mineral water. The details are as follows: table tennis players start the game at the same time. Experiment 2 uses the same method to ensure that all collected parameters are at the same point in time. The same diet was provided during the test on day 2 and on 
TABLE 1: The basic situation of the tested table tennis players.

\begin{tabular}{lccc}
\hline Index & Male & Female & Range \\
\hline Age & $19-23$ & $18-21$ & $20.5 \pm 0.3$ \\
Body weight $(\mathrm{kg})$ & $75 \pm 4.6$ & $60 \pm 3.5$ & $55.6 \pm 3.56$ \\
Height $(\mathrm{cm})$ & $175 \pm 5$ & $166 \pm 5$ & $170.3 \pm 4.38$ \\
Training time & $6.2 \pm 1.3$ & $5.9 \pm 1.1$ & $5.7 \pm 0.8$ \\
\hline
\end{tabular}

the first day before the test. Before the test and for 1 week during the test, smoking, drinking, drinking coffee, and using central stimulants or tranquilizers are prohibited. Table tennis players in the observation group supplemented with $0.3 / \mathrm{kg}$ branched-chain amino acids, and dissolved the branched-chain amino acid powder in $150 \mathrm{~mL}$ of grape juice to mask the smell. An appropriate amount of starch was dissolved in $250 \mathrm{~mL}$ of mineral water, and starch-containing capsules were added to the control group as a placebo.

3.2.2. Sports Plan. Two one-hour table tennis matches will be held in two days. The table tennis match will last for half an hour and then rest for ten minutes. First, do a 5-10 minute warm-up, and then, start the exercise ability test. The test is divided into the upper and lower halves for 30 minutes each, with a 10-minute rest in between. The test consists of 10 sets of the same 60 -minute exercise. The 60-minute exercise includes the following: 5 minutes of lateral sliding, 5 minutes of reentry, 30 minutes of table tennis competition, and 10 minutes of 500 meters. The exercise routines are single and bipedal jumps 20 times, 5 -minute sprint, 5-minute continuous bounce, 5-minute pull ups. The photoelectric timer is used to record the time required by table tennis players every 20 meters. In the experiment, because the physical fitness of men and women is different but because they are training together, we have carried out the training to a certain degree, so their exercise program is the same.

3.3. Table Tennis Player's Biological Index Detection and Athletic Ability Determination. On the day of the test, blood was drawn from table tennis players by venipuncture before breakfast and immediately after the test and biological indicators were measured. The blood sample was collected in an EDTA.k3 vacuum container, and the plasma was extracted at $1500 \times \mathrm{g}$ through an Epd510 centrifuge and stored at $-50^{\circ} \mathrm{C}$ for later use. Instructions for the biotic drug kit measure the concentration of the patient's plasma branched-chain acetamido acid enzyme at the same time, and the measurement method is strictly in accordance with the instructions for the drug kit. The plasma radiation concentration of free arginine can be determined by various fluorescence irradiation analyses. The average concentration of glycerol, free saturated fatty acid (near), and lactate in the patient's plasma (nh3) was directly measured with the Hitachi 7020 automatic biochemical analyzer. Use a pulse meter to measure the number of heart beats per minute of a table tennis player, use a thermometer to measure the table tennis player's temperature after exercise, and use a stethoscope to measure the table tennis player's breathing rate; catalase $\mathrm{CAT}$ is measured with hydrogen peroxide.

The ep206-p random reaction time mobile analyzer is used to determine the random reaction time (measurement method: randomly set the reaction $n=50$ times, and quickly get on and off the car according to the reaction light and stimuli that appear in the random condition to average the rapid response). Time average fast-moving reaction time and correct coupling response are the main indicators of this test. Use the EP708A attention allocator to measure the ability to allocate attention (determination method: the subject is tested under separate acousto-optic stimulation and each measurement combines each for 1 minute and record the $Q$ value). Cognitive performance indicators include the following: endurance index, fatigue after exercise, and exercise reaction time.

3.4. Data Statistics Method. The test measurement results are all expressed by the formula of measuring the average error standard value error. The data analysis of the test results can use the multiple ANOVA method program in the spspss18.0 software version to perform a single multivariate analysis of variance. If the difference is significant, announce. Roche method can also be used for multivariate comparison analysis. $p>0.05$ means that the clinical difference is not significant, $p<0.05$ means that the clinical difference is significant, and $p<0.01$ means that the clinical difference is extremely significant.

\section{Experimental Results of the Influence of Branched-Chain Amino Acids on Exercise Ability}

4.1. Analysis of the Experimental Results of the Effects of Supplementing Branched-Chain Amino Acids on Exercise Ability. The results of the study showed that the time to exhaustion of table tennis players in the observation group supplemented with branched-chain amino acids was $(48.43 \pm 5.8 \mathrm{~min})$ and the time to exhaustion of table tennis players in the control group was (36.12 $\pm 7.55 \mathrm{~min})$. Compared with the training group, the rate of ....... The rate of exhaustion and delay of rats in the BCAA group was $23.05 \%$, with a significant difference $(p<0.05)$. The heart rate of athletes in the observation group supplemented with branched-chain amino acids was 72 beats per minute after exercise, and the heart rate of athletes in the control group was 83 beats per minute. There was a statistical difference between the two groups $(p<0.05)$. After exercise, the blood glucose of the observation group supplemented with branched-chain amino acids was significantly higher than that of the control group, indicating that branched-chain amino acids can improve the blood sugar of athletes. The specific information on the blood sugar level, heart rate, and exhaustion time of the two groups of table tennis players after the game is shown in Table 2.

The results of the study showed that the fatigue index of athletes in the observation group supplemented with branched-chain amino acids was lower than that in the control group and there was a statistical difference $(p<0.05)$. In 
TABLE 2: Specific information on the blood sugar level, heart rate, and exhaustion time of the two groups of table tennis players after the game.

\begin{tabular}{lcccc}
\hline Data value & Blood sugar level & Heart rate & Exhausted time & Breath rate \\
\hline Observation group & $145.8 \pm 7.21$ & $70 \pm 3.5$ & $48.43 \pm 5.86$ & $63.32-68.35$ \\
Control group & $148.4 \pm 6.69$ & $80 \pm 5.2$ & $36.12 \pm 7.55$ & $75.32-80.28$ \\
$T$ & 5.826 & 6.472 & 6.365 & 7.214 \\
\hline
\end{tabular}
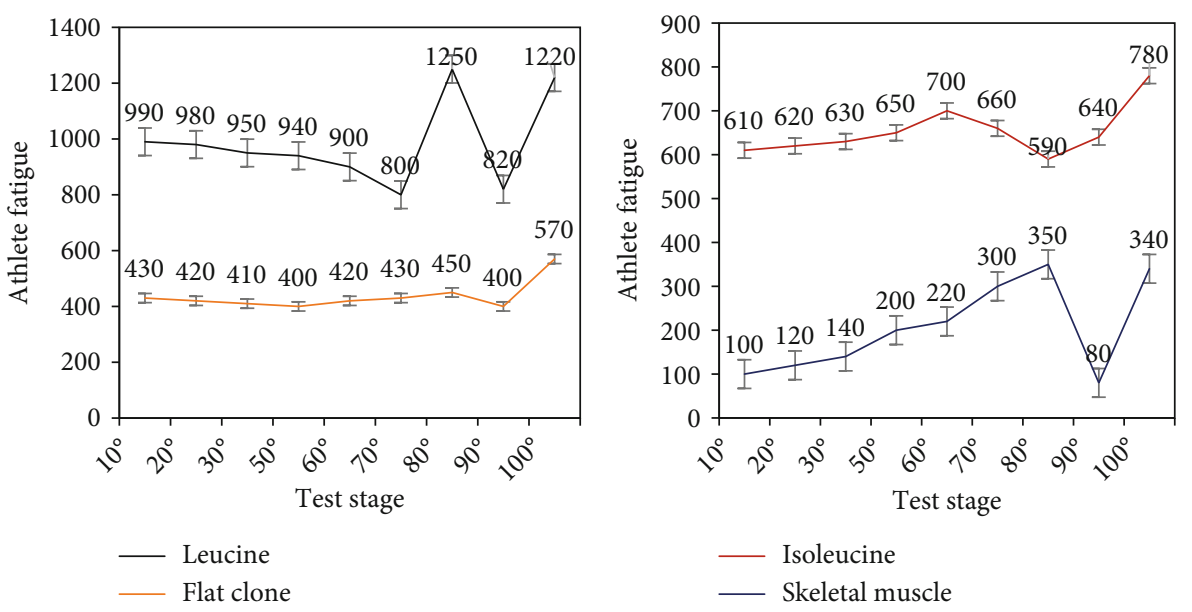

Figure 7: The effect of supplementing branched-chain amino acids on the fatigue of table tennis players.

table tennis, branched-chain amino acids are important amino acids involved in energy supply during sustained exercise. The decomposition of branched-chain amino acids in muscle is very active. Compared with other amino acids, branched-chain amino acids can quickly and completely rotate amino acids. Its molecular efficiency is much higher than the molecular efficiency of the other three amino acids in the oxidation reaction to generate molecular ATP. All amino acid molecules completely oxidize leonine, isoleucine, and non-isoleucine, and the molecular efficiency of the oxidation reaction to produce the molecule ATP (Adenosine triphosphate) is much higher than those of the other three amino acids, leonine, and isoleucine. Each molecule is completely oxidized. When a table tennis player is quiet, the process of amino acid synthesis oxidase in the branch chain can provide about $18 \%$ of the total physiological energy consumption of human skeletal muscle at the same time. Under certain special conditions, such as hunger, breastfeeding, and exercise, the increased oxidative supply of branched-chain amino acids is an important energy source in the body. Studies have shown that during fasting, the ability of muscles to oxidize branched-chain amino acid transaminase keno acid can be increased by 4-7 times. After table tennis, the concentration of branched-chain amino acids in the plasma decreased significantly, while the exercise group supplemented with branched-chain amino acids increased the exercise time and reduced the response to fatigue. The research results show that supplementation of branched-chain amino acids can effectively reduce the fatigue of table tennis players. The relevant data is shown in Figure 7.
It can be seen from the data in Figure 7 that supplementation of branched-chain amino acids can effectively reduce the fatigue of table tennis players. After supplementation of branched-chain amino acids, the fatigue index of the athletes in the observation group after the game is $(7.5 \pm 1.2)$ and the fatigue index of the control group is (11.2 \pm 1.5$)$.

The results of the study showed that the endurance score of table tennis players in the observation group supplemented with branched-chain amino acids was significantly higher than that in the control group and there was a statistical difference between the two groups $(p<0.05)$. Compared with those of the control group and the observation group, the BU content of table tennis players is reduced. One of the reasons is the supplementation of branched-chain amino acids. Glycosylase provides abundant raw materials for liver function, so that sugar enhances the blood synthetic function and maintains sugar levels. After the blood sugar level is constant, the energy released by the blood sugar is transferred to the muscle tissue for oxidation and supplied to the exercise tissue. The decomposition rate of this protein decreases, the released NHs decrease, and the production of urea decreases. The supplementation of branched-chain amino acids can reduce the decomposition of hemoglobin caused by strenuous exercise, which is beneficial to maintain the athletic ability of table tennis players. Studies have found not only that table tennis reduces the concentration of branched-chain amino acids in the serum but also that the content of branched-chain amino acids in muscle tissues has also decreased significantly, indicating that table tennis accelerates the decomposition of muscle proteins and the release of aromatic amino acids. Branched-chain amino acid 

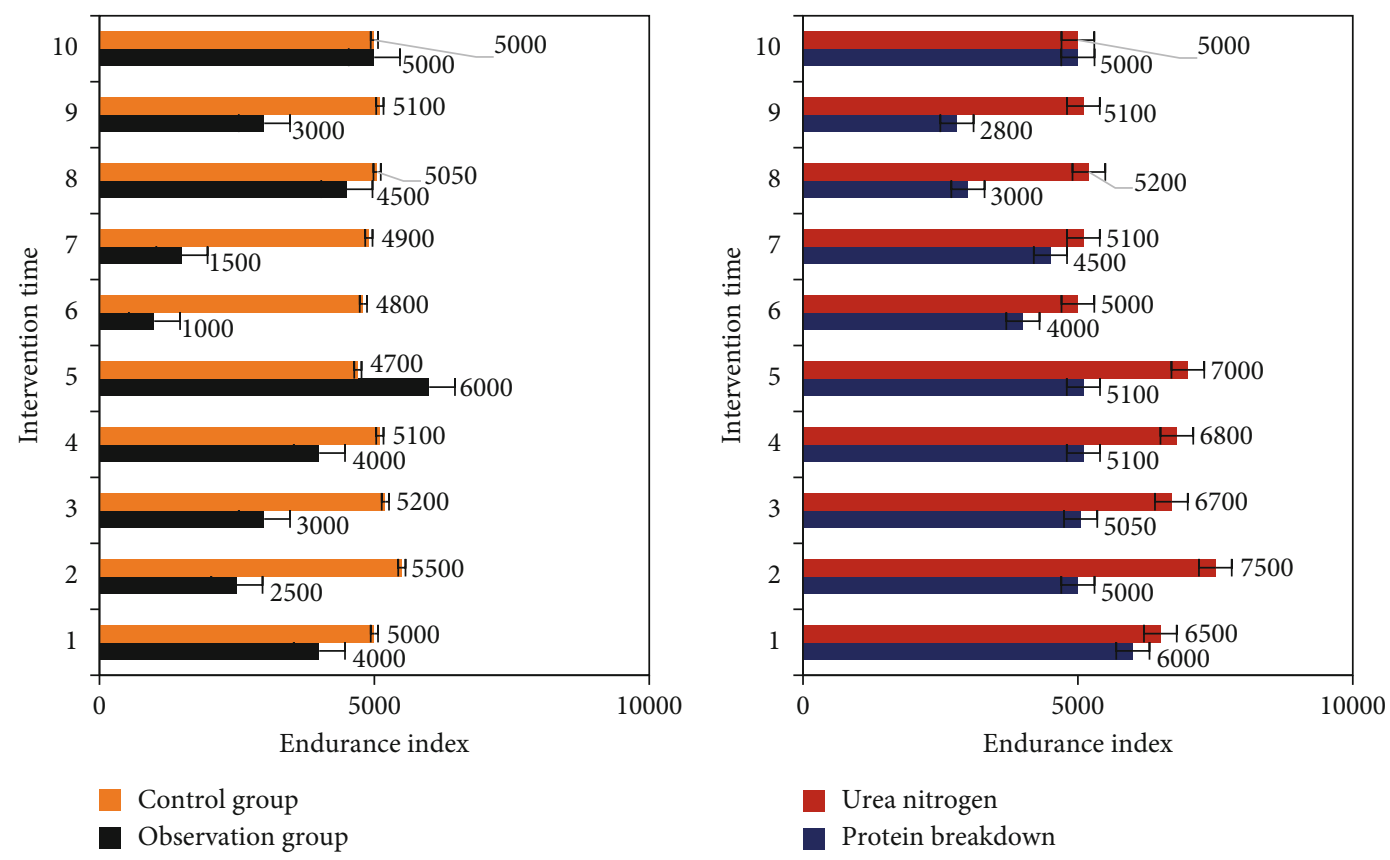

Figure 8: The specific effect of supplementing branched-chain amino acids on the endurance index and reaction speed of table tennis players.

supplements can improve this situation, thereby improving athletes' endurance and reaction speed. The research results show that supplementation of branched-chain amino acids can improve the endurance index and reaction speed of table tennis players. The specific data is shown in Figure 8.

From the data in Figure 8, it can be seen that supplementation of branched-chain amino acids can improve the endurance index and reaction speed of table tennis players. The endurance index of the observation group supplemented with branched-chain amino acids is (28.4 \pm 4.8$)$, and that of the control group is $(19.6 \pm 3.7)$. The reaction ability index of the observation group was $(18.4 \pm 4.2)$, and that of the control group was $(12.3 \pm 2.8)$.

As shown in Table 3, after one exercise, the plasma testosterone concentration of the athletes who used the placebo group decreased significantly $(p<0.05)$, while the athletes who used BCAA showed a slight upward trend but not significant. After long-term supplementation of BCAA exercise, $T$ increased significantly $(p<0.05)$, while the placebo group decreased slightly after exercise, and the $T$ value of the BCAA group after exercise was significantly higher than that of the placebo group $(p<0.05)$.

As shown in Table 4, the plasma cortisol value of onetime exercise or long-term exercise is significantly higher than that of the resting state, while the plasma cortisol value of long-term exercise and supplementing with BCAA in the resting state is significantly lower than that of placebo group $(p<0.05)$; there was no significant difference between the two groups immediately after exercise. After using BCAA, the plasma testosterone concentration of athletes before exercise is $767 \pm 134$, and after exercise, it is $758 \pm 295$, which is still different from the difference without taking it.
TABLE 3: Changes in plasma testosterone concentration before and after one-time and long-term supplementation of BCAA athletes.

\begin{tabular}{lcccc}
\hline \multirow{2}{*}{ Exercise mode } & \multicolumn{2}{c}{ Placebo } & \multicolumn{2}{c}{ BCAA } \\
& exercise & $\begin{array}{c}\text { After } \\
\text { exercise }\end{array}$ & $\begin{array}{c}\text { Before } \\
\text { exercise }\end{array}$ & $\begin{array}{c}\text { After } \\
\text { exercise }\end{array}$ \\
\hline $\begin{array}{l}\text { One-time } \\
\text { exercise }\end{array}$ & $984 \pm 109$ & $654 \pm 340$ & $767 \pm 134$ & $758 \pm 295$ \\
$\begin{array}{l}\text { Long-term } \\
\text { exercise }\end{array}$ & $695 \pm 176$ & $681 \pm 254$ & $902 \pm 143$ & $1098 \pm 345$ \\
\hline
\end{tabular}

As can be seen in Table 5, the $T / C$ ratio of the placebo group and the BCAA group decreased significantly immediately after a one-time exercise $(p<0.05)$. Although the value of the BCAA group decreased but not significantly, the value of the long-term exercise BCAA group was better and it was significantly higher than that of the placebo group $(p<0.05)$.

4.2. Analysis of the Influence of Supplementing BranchedChain Amino Acids on the Physiological Index of Table Tennis Players. Studies have found that branched-chain amino acids can significantly reduce the level of malondialdehyde (MDA) of table tennis players and protect the levels of acetyl glutathione super peroxidase (GSH-PX) and hydrochloric superoxide dismutase (sod) dismutase activity and can be used to significantly increase the content of acetyl glutamate and acetyl aspartic acid in serum myocardium and increase the level of p-phenylalanine in serum and solution myocardium. Therefore, supplementing table tennis players with amino acids has a certain protective effect on ischemic heart disease. The results show that branched-chain amino acids after exercise will also have a 
TABLE 4: Effects of one-time and long-term BCAA supplementation on plasma cortisol concentration before and after exercise.

\begin{tabular}{lcccc}
\hline Exercise mode & Placebo & & & BCAA \\
& Before exercise & After exercise & Before exercise & After exercise \\
\hline One-time exercise & $16.98 \pm 2.9$ & $23.58 \pm 3.2$ & $23.05 \pm 6.7$ & $25.91 \pm 5.2$ \\
Long-term exercise & $21.5 \pm 4.9$ & $28.06 \pm 6.5$ & $11.89 \pm 6.4$ & $23.57 \pm 9.5$ \\
\hline
\end{tabular}

TABLE 5: Changes of the plasma testosterone/cortisol ratio in one-time and long-term supplementation of BCAA.

\begin{tabular}{|c|c|c|c|c|}
\hline \multirow{2}{*}{ Exercise mode } & \multicolumn{2}{|c|}{ Placebo } & \multicolumn{2}{|c|}{ BCAA } \\
\hline & Before exercise & After exercise & Before exercise & After exercise \\
\hline One-time exercise & $50.9 \pm 35.6$ & $28.64 \pm 13.2$ & $35.6 \pm 20.9$ & $29.21 \pm 19.8$ \\
\hline Long-term exercise & $36.21 \pm 18.79$ & $25.6 \pm 11.2$ & $71.2 \pm 35.4$ & $45.4 \pm 19.5$ \\
\hline
\end{tabular}
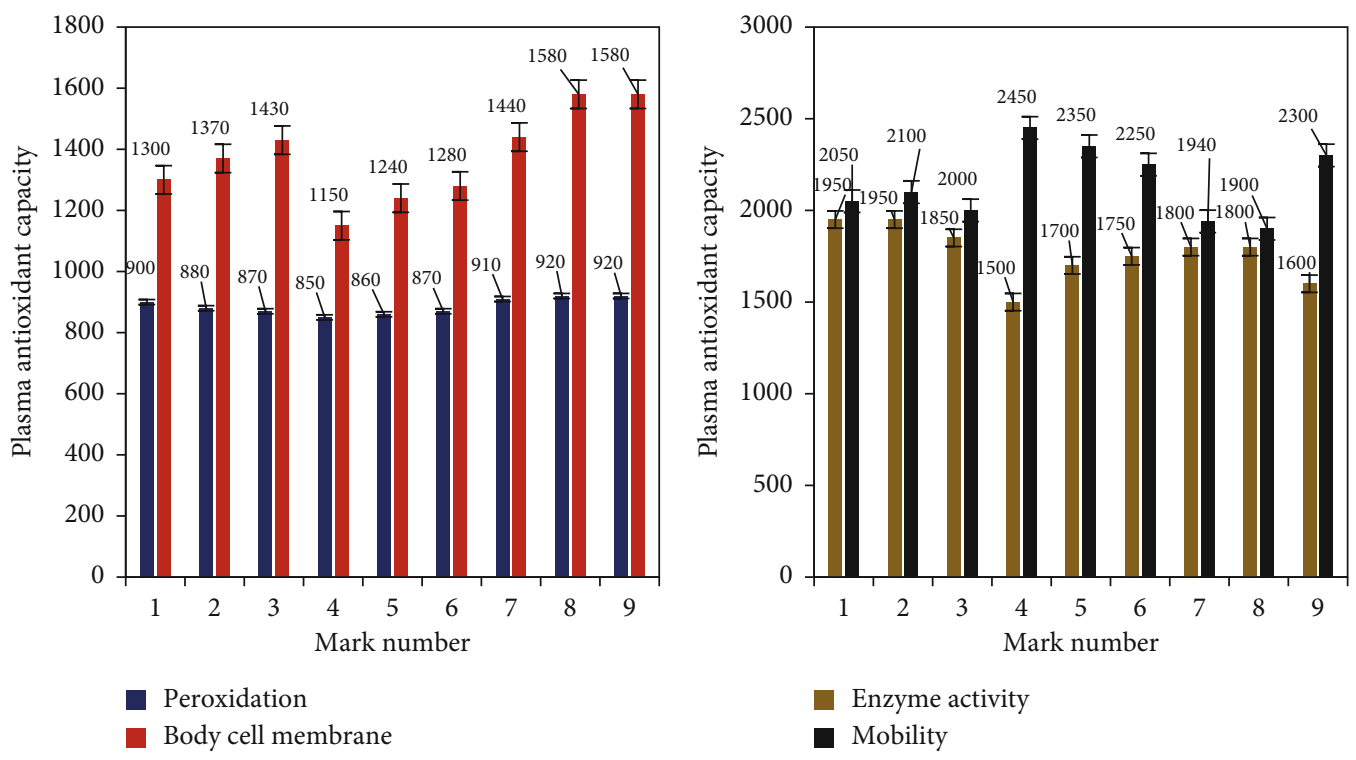

Figure 9: The effect of branched-chain amino acids on the plasma antioxidant capacity of athletes in table tennis.

certain impact on the activities of free radicals and antioxidant enzymes. There is a possibility of influence on the transcription or translation process of chain amino acids and sod. It is based on the influence of the gene expression of antioxidant enzymes in the body, the influence of valise, leonine and protein synthesis, and the human body sod in the plasma, so the oxidation level declines and the athlete's state declines. The fatigue of table tennis players after exercise has a lot to do with the calcium concentration inside and outside the cell. The accumulation of calcium in cells will affect the recovery of exercise fatigue. Branched-chain amino acids can reduce the calcium load in cells and hinder the formation of free radicals. Enzyme free radicals have a certain degree of protection and can hinder the oxidation reaction in the body. The research results show that branched-chain amino acids can improve the plasma antioxidant capacity of athletes in table tennis. The relevant data are shown in Figure 9. This proves through related experiments that we should not consume too much branched-chain amino acids, which will cause the reduction of our life span and the aging of various functions of the body.

It can be seen from the data in Figure 9 that branchedchain amino acids can improve the plasma antioxidant capacity of table tennis players in table tennis. Compared with the control group, the table tennis players' plasma antioxidant capacity in the observation group supplemented with amino acids increased by $36.2 \%$. Through the above picture, it can be clearly described as an appropriate supplementation of branched-chain amino acids, which can greatly enhance the athletic ability of athletes.

The research in this article found that in table tennis, branched-chain amino acids can not only be used as energy supply substances but also have the effect of regulating protein synthesis. Branched-chain amino acids are thought to have insulin-like effects and are an important part of muscle fibers. The research in this article shows that leonine is an important amino acid in muscle composition. Ingested leonine is transferred to muscle cells and then converted into 

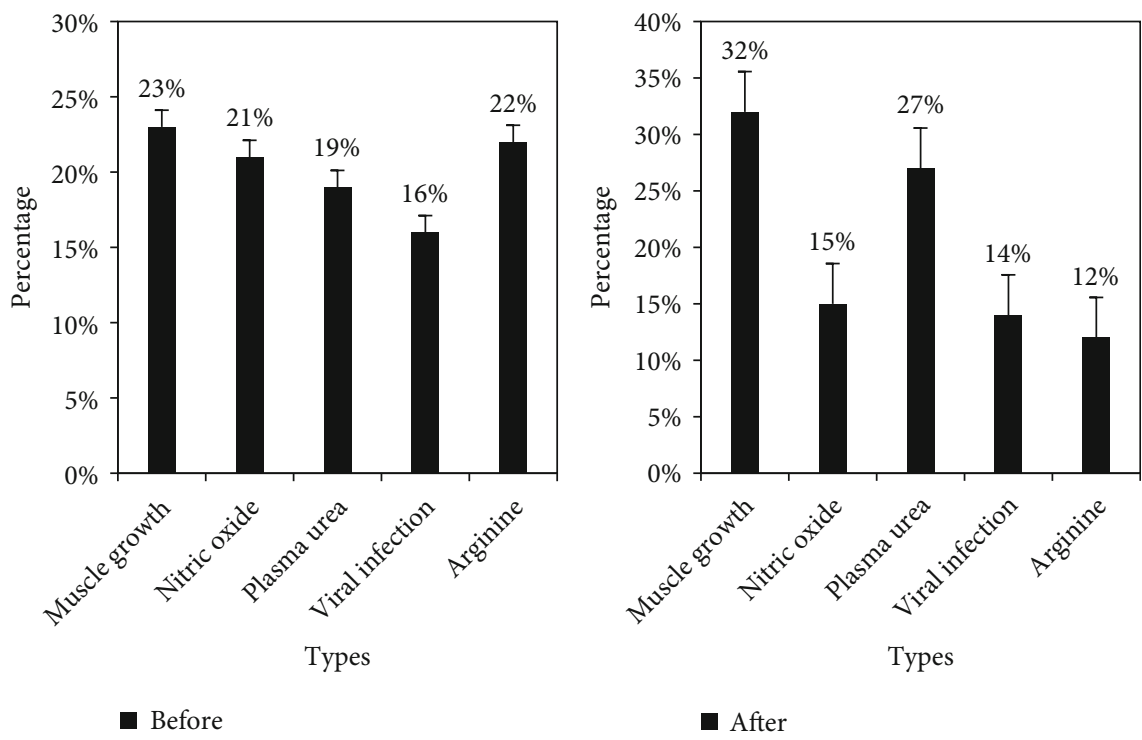

FIgURE 10: The effect of supplementing branched-chain amino acids on the metabolism of table tennis players.

ketones. Branched-chain amino acids are used to generate intermediate products in the tricarboxylic acid cycle. In the process of excessive energy consumption, appropriate supplementation of branched-chain amino acids can reduce the decomposition of the body's own protein. Since the nitrogen metabolism of branched-chain amino acids is carried out in the form of nontoxic glutamine transport, it can reduce the excessive production of serum urea nitrogen, that is, reduce the burden on the liver and kidneys, reduce blood urea, and thereby reduce blood flow ammonia levels. Tennis players supplemented with branched-chain amino acids before the game can also prevent serotonin from entering the brain and prevent central nervous system fatigue. At the same time, supplementing branched-chain amino acids in table tennis training can promote muscle protein synthesis and prevent muscle protein degradation. Speed up the metabolism of athletes. The research results show that supplementation of branched-chain amino acids can improve the metabolism of table tennis players. The specific data is shown in Figure 10.

It can be seen in Figure 10 that supplementation of branched-chain amino acids can increase the metabolism of table tennis players. We can get relevant results by adding and subtracting each data compared with those of the control group; the metabolism of the observation group of supplemented branched-chain amino acids increased by $28.5 \%$, thus accelerating the physical recovery of table tennis players.

\section{Conclusions}

(1) Table tennis is a very popular sport. In this sport, athletes need to constantly run, beat, and maintain concentration. Therefore, this sport is also a sport that consumes physical energy. Branched-chain amino acids are the basic constituents of proteins that are closely related to life activities. It is closely related to athletic ability. Studies have found that branched-chain amino acids can delay the time of exhaustion for table tennis players and at the same time can improve athletes' explosive power

(2) The results of the study showed that after the supplementation of branched-chain amino acids, the fatigue index of the athletes in the observation group was $(7.5 \pm 1.2)$, the fatigue index of the control group was (11.2 \pm 1.5$)$, the endurance index of the observation group was $(28.4 \pm 4.8)$, that of the control group was $(19.6 \pm 3.7)$, the reaction ability index of the observation group was $(18.4 \pm 4.2)$, and that of the control group was $(12.3 \pm 2.8)$. At the same time, the athletes' plasma antioxidant capacity and metabolism level of the observation group were significantly higher than those of the control group. Therefore, it can be seen that supplementing branched-chain amino acids can effectively improve the athletic ability of table tennis players. Through this experiment, we will have a certain understanding that it is impossible to replenish energy to the best state immediately after the athlete exercises. We also need to consider whether this thing will cause a certain amount of damage to the athlete's body, to ensure the physical health of our athletes

(3) This study found that branched-chain amino acids can improve the plasma antioxidant capacity of table tennis players. Compared with the control group, the table tennis players' plasma antioxidant capacity in the observation group supplemented with amino acids increased by $36.2 \%$. At the same time, branched-chain amino acid supplementation can improve table tennis. Compared with the control group, the metabolism level of the ball players increased by $28.5 \%$ in the observation group supplemented with branched-chain amino acids, thus 
accelerating the physical recovery of table tennis players. Branched-chain amino acids can improve the plasma antioxidant capacity of athletes in table tennis, can improve athletes' athletic ability, and can help them to replenish energy in time after consuming energy. However, there are still some serious problems in the research of the article. Some of the influencing factors of the experiment cannot be ruled out and affect the results of the experiment. This needs to be supplemented in subsequent studies. Through the research on the athletic ability of table tennis players, we can apply it to other sports accordingly. This supplementation of branchedchain amino acids or other energy supplements can improve the physical fitness of athletes

\section{Data Availability}

All the data used are given in the paper.

\section{Conflicts of Interest}

The author declares that they have no conflicts of interest.

\section{Acknowledgments}

This work was supported by the teaching reform of Sichuan Minzu College (J18029): pilot project of comprehensive reform of physical education application-oriented specialty.

\section{References}

[1] A. Morales, N. Arce, and M. Cota, "Effect of dietary excess of branched-chain amino acids on performance and serum concentrations of amino acids in growing pigs," Journal of Animal Physiology and Animal Nutrition, vol. 100, no. 1, pp. 39-45, 2016.

[2] A. D. Osmond, D. J. Directo, and M. L. Elam, "The effects of leucine-enriched branched-chain amino acid supplementation on recovery after high-intensity resistance exercise," International Journal of Sports Physiology and Performance, vol. 14, no. 8, pp. 1-25, 2019.

[3] X. Shi, X. Wei, and X. Yin, "Hepatic and fecal metabolomic analysis of the effects of Lactobacillus rhamnosus GG on alcoholic fatty liver disease in mice," Journal of Proteome Research, vol. 14, no. 2, pp. 1174-1182, 2015.

[4] W. C. Kephart, T. D. Wachs, and R. M. Thompson, "Ten weeks of branched-chain amino acid supplementation improves selected performance and immunological variables in trained cyclists," Amino Acids, vol. 48, no. 1, pp. 779-789, 2016.

[5] Z. Wan, Y. Dong, Z. Yu, H. Lv, and Z. Lv, "Semi-supervised support vector machine for digital twins based brain image fusion," Frontiers in Neuroscience, vol. 15, article 705323, 2021.

[6] M. Hu, Y. Zhong, S. Xie, H. Lv, and Z. Lv, "Fuzzy system-based medical image processing for brain disease prediction," Frontiers in Neuroscience, vol. 965, 2021.

[7] W. Chen, Y. Y. Jiang, and J. P. Wang, "Effect of flaxseed on the fatty acid profile of egg yolk and antioxidant status of their neonatal offspring in Huoyan geese," Animal, vol. 9, no. 11, pp. 1749-1755, 2015.
[8] S. I. Yokota, M. Ando, and S. Aoyama, "Leucine restores murine hepatic triglyceride accumulation induced by a low-protein diet by suppressing autophagy and excessive endoplasmic reticulum stress," Amino Acids, vol. 48, no. 4, pp. 1013-1021, 2016.

[9] W. Wambacq, G. Rybachuk, and I. Jeusette, "Fermentable soluble fibres spare amino acids in healthy dogs fed a low-protein diet," BMC Veterinary Research, vol. 12, no. 1, pp. 1-10, 2016.

[10] S. Parivash, E. Mansoor, and K. Jahangir, "The effect of branched chain amino acids supplementation (BCAA) on muscle damage and the indicators of fatigue in soccer players," Atherosclerosis Supplements, vol. 32, no. 10, pp. 135-136, 2018.

[11] R. U. Ruiz-Margáin and S. L. R.-T. Macías-Rodríguez, "Effect of a high-protein, high-fiber diet plus supplementation with branched-chain amino acids on the nutritional status of patients with cirrhosis," Revista de Gastroenterología de México (English Edition), vol. 83, no. 1, pp. 9-15, 2018.

[12] Q. Jun, Z. Dong, and Y. He, "Research progress on the mechanism of supplementation of branched-chain amino acids on the effects and safety of athletes in different sports," Sports Research, vol. 37, no. 6, pp. 88-92, 2016.

[13] C. R. Hong, G. W. Lee, and H. D. Paik, "Influence of biopolymers on the solubility of branched-chain amino acids and stability of their solutions," Food Chemistry, vol. 239, no. 15, pp. 872-878, 2018.

[14] D. Chen, W. C. Vong, and S. Q. Liu, "Effects of branched-chain amino acid addition on chemical constituents in lychee wine fermented with saccharomyces cerevisiae," International Journal of Food Science \& Technology, vol. 50, no. 12, pp. 25192528, 2016.

[15] L. Peilin, "Brief analysis on the nutrition of table tennis players," Youth Sports, vol. 10, no. 7, pp. 107-108, 2018.

[16] H. Wu and Z. Dongmei, "Effect of amino acid compound preparation on central fatigue after high-intensity interval exercise," China Tissue Engineering Research, vol. 22, no. 20, pp. 3150-3154, 2018.

[17] D. Sheikholeslami-Vatani and S. Ahmadi, "Effect of oral branched-chain amino acid supplementation prior to resistance exercise on metabolic hormones, plasma amino acids, and serum indices of muscle damage in the recovery period," Topics in Clinical Nutrition, vol. 31, no. 4, pp. 346-354, 2016. 